\title{
Extraction of series resistance and mobility degradation parameter in MOSFETs using iterative method
}

\author{
Noureddine Maouhoub ${ }^{1}$, Khalid Rais ${ }^{2}$ \\ ${ }^{1}$ Laboratory of Electronics, Signal Processing and Physical Modeling, Department of Physics, Ibn Zohr University, \\ Faculty of Sciences, Morocco \\ ${ }^{2}$ Laboratory of Electronics, Instrumentation and Energy, Faculty of Science, Chouaib Doukkali University, \\ Department of Physics, Morocco
}

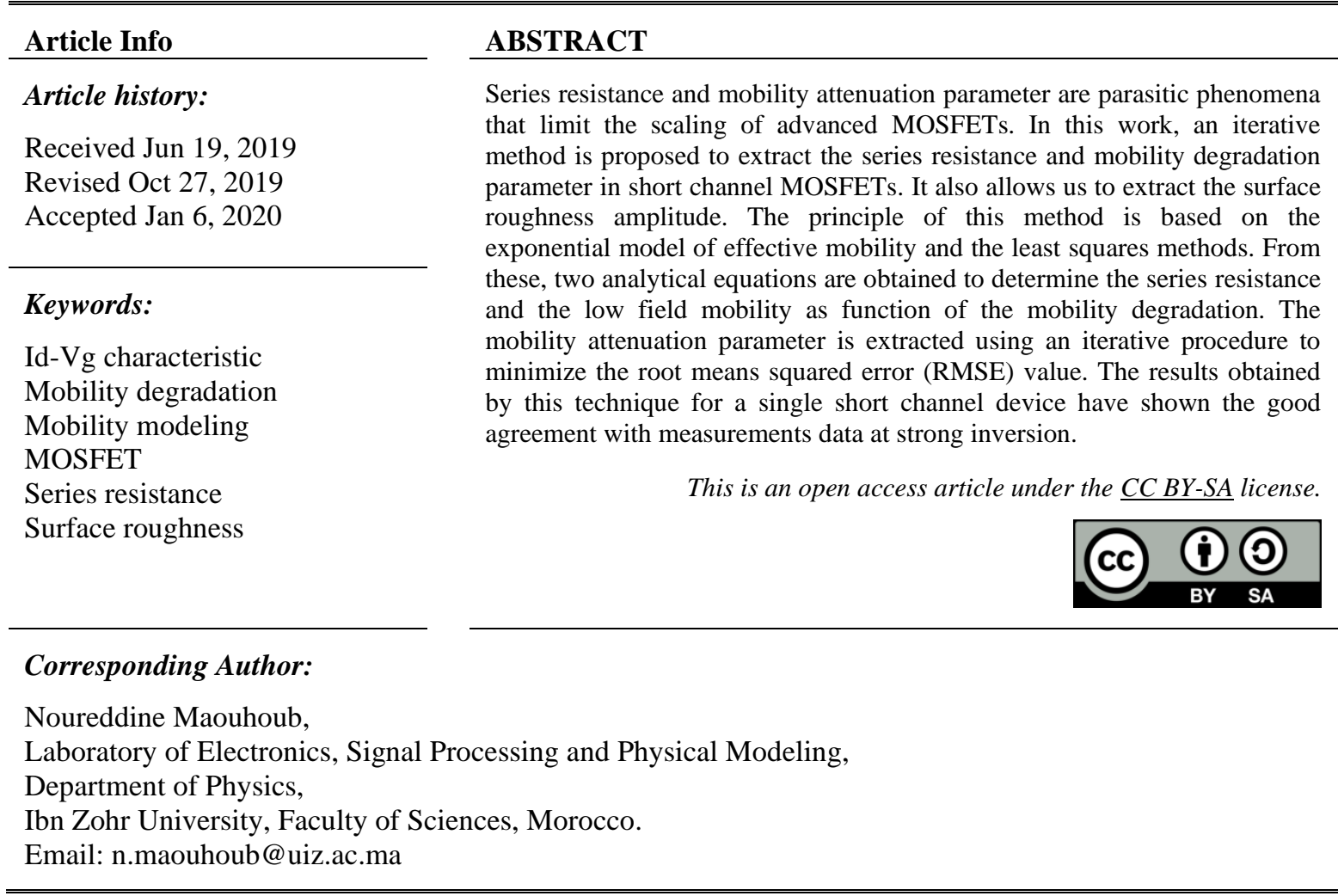

\section{INTRODUCTION}

Mobility degradation phenomena and parasitic source-and-drain series resistance are two parameters of special importance for MOSFET characterization. These two parameters limit scaling of advanced devices and produce effects on the device's transfer drain current-gate voltage characteristics. Accurate modeling and extraction of these parameters have a most importance for MOSFET.

According the literature, several methods and procedures have been proposed to extract the series resistance and the mobility degradation parameters [1-15]. Another methods were proposed to extract these parameters using a novel test structures, such as the multifinger MOSFET [16-17], the FinFET [18] and the amorphous MOSFET [19-20]. Recently, in works [21], a review of DC extraction methods for MOSFET parasitic series resistance and mobility degradation model parameters has been presented. In the previous work, Ortiz et al have presented and critically compared a total of eighteen extraction procedures; some of these methods use several devices with different mask channel lengths and other methods need a single device but under different drain biases. Rodriguez-Davila et al proposed a new integration-basesed procedure to extract the mobility enhancement factor, and the series resistance of thin- film MOSFETs [22].

In this paper we present an iterative method based on exponential model of mobility and analytical approach using least squares method. We formulate two analytical expressions as function of the mobility attenuation parameter. This parameter is then extracted iteratively at the minimum of the root means squared 
error. The advantage of this method that is used only a single test device and one drain biase and alow us also to extract the surface roughness amplitude.

The paper is organized as follows: Section 2 presents the modeling of the drain current using the model of effective mobility and we formulate the extraction technique. The validation of the proposed method using experiment data of single device is shown in section 3. Finally, the paper is closed by the conclusion of section 4 .

\section{MODELING AND EXTRACTION TECHNIQUE}

\subsection{Drain current modeling}

The drain current $\mathrm{I}_{\mathrm{d}}$, at very small drain bias, can be expressed in terms of intrinsic voltages as:

$$
\mathrm{I}_{\mathrm{d}}=\frac{\mathrm{W}}{\mathrm{L}} \mu_{\mathrm{eff}} \mathrm{C}_{\mathrm{ox}}\left(\mathrm{V}_{\mathrm{G}}-\mathrm{V}_{\mathrm{t}}\right) \mathrm{V}_{\mathrm{D}}
$$

Where $\mathrm{W}$ is the channel width, $\mathrm{L}$ is the channel length, Cox is the oxide capacitance, $\mu_{\mathrm{eff}}$ is the effective free-carrier mobility, $V_{G}$ is the intrinsic gate voltage, $V_{D}$ the intrinsic drain voltage and $V_{t}$ is the threshold voltage. If the source-and-drain series resistance is significant, the device's intrinsic gate and drain voltages are:

$$
\begin{aligned}
& V_{G}=V_{g}-I_{d} \cdot \frac{R_{s d}}{2} \\
& V_{D}=V_{d}-I_{d} \cdot R_{s d}
\end{aligned}
$$

Where $V_{g}$ and $V_{d}$ are the externally applied gate and drain voltages, respectively, $R_{s d}$ is the total source-and-drain series resistance as shown in Figure 1.

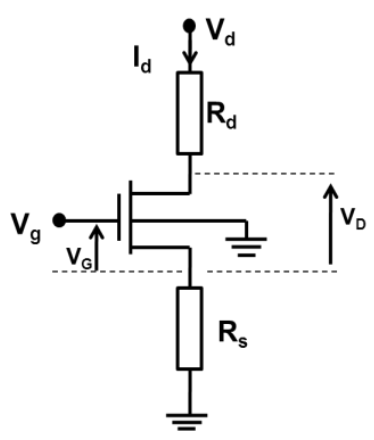

Figure 1. Intrinsic and externally applied voltages in MOSFET

The model of variation mobility with effective field considers that the attenuation of effective mobility is particularly due to Surface Roughness Scattering [4]. This work generalized all the classical models $[4,10,12]$, and provided a physical meaning to different used parameters.

The effective mobility is given by the exponential model in strong inversion $[4,12]$ :

$$
\mu_{\text {eff }}=\mu_{0} \cdot \exp \left(-\theta\left(V_{G}-V_{t}\right)\right)
$$

Where,

$$
\theta=\frac{\eta \cdot \beta \cdot \Delta \cdot \mathrm{C}_{\mathrm{ox}}}{\varepsilon_{\mathrm{si}}}
$$


$\theta$ is the intrinsic attenuation coefficient of mobility, $\eta$ is constant parameter equal to 0.5 for electrons and 0.33 for holes [4], $\beta=\mathrm{q} / \mathrm{kT}$ is the inverse of the thermal potential, $\varepsilon_{\mathrm{si}}$ silicon permittivity, $\Delta$ is the surface roughness amplitude, $\mu_{0}$ represents the low-field mobility.

Assuming that $V_{g}-V_{t}>I_{d} \cdot R_{s d} / 2$, and substitution of (4) in (1) yields:

$$
I_{d}=K \cdot \frac{V_{g}-V_{t}}{\exp \left(\theta\left(V_{g}-V_{t}\right)\right)+\frac{K \cdot R_{s d}}{V_{d}}\left(V_{g}-V_{t}\right)}
$$

Where,

$$
\mathrm{K}=\frac{\mathrm{W}}{\mathrm{L}} \cdot \mu_{0} \cdot \mathrm{C}_{\mathrm{ox}} \cdot \mathrm{V}_{\mathrm{d}}
$$

\subsection{Extraction method}

The procedure is based on calculating the function $\mathrm{R}\left(\mathrm{V}_{\mathrm{g}}\right)$ :

$$
R\left(V_{g}\right)=\frac{V_{g}-V_{t}}{I_{d}}=\frac{V_{g t}}{I_{d}}=\frac{1}{K} \cdot \exp \left(\theta V_{g t}\right)+\frac{R_{s d}}{V_{d}} \cdot V_{g t}
$$

The problem consists to minimize the follow objective function:

$$
\mathrm{S}=\sum_{\mathrm{i}=1}^{\mathrm{N}}\left(\mathrm{R}_{\mathrm{i}, \mathrm{th}}\left(\mathrm{V}_{\mathrm{gti}}, \mathrm{K}, \mathrm{R}_{\mathrm{sd}}\right)-\mathrm{R}_{\mathrm{i}, \text { exp }}\right)^{2}
$$

$\mathrm{I}_{\mathrm{i}, \mathrm{cal}}$ is the theoretical characteristic that can be rewritten using the (1) :

$$
\mathrm{R}_{\mathrm{i}, \mathrm{th}}\left(\mathrm{V}_{\mathrm{gti}}\right)=\frac{1}{\mathrm{~K}} \cdot \mathrm{EXP}_{\mathrm{i}}+\frac{\mathrm{R}_{\mathrm{sd}}}{\mathrm{V}_{\mathrm{d}}} \cdot \mathrm{V}_{\mathrm{gti}}
$$

Where EXPi is given by:

$$
\mathrm{EXP}_{\mathrm{i}}=\exp \left(\theta . \mathrm{V}_{\mathrm{gti}}\right)
$$

$\mathrm{R}_{\mathrm{i} \text { exp }}$ and $\mathrm{V}_{\mathrm{gti}}$ are the measured $\mathrm{R}$ and voltage at the ith point and $\mathrm{N}$ is the number of measured data points. For minimizing the objective function $\mathrm{S}$, we must solve the following system:

$$
\left\{\begin{array}{l}
\frac{\partial \mathrm{S}}{\partial(1 / \mathrm{K})}=2 \sum_{\mathrm{i}=1}^{\mathrm{N}} \frac{\partial \mathrm{R}_{\mathrm{i}, \text { th }}}{\partial(1 / \mathrm{K})}\left(\mathrm{R}_{\mathrm{i}, \text { th }}-\mathrm{R}_{\mathrm{i}, \exp }\right)=0 \\
\frac{\partial \mathrm{S}}{\partial \mathrm{R}_{\mathrm{sd}}}=2 \sum_{\mathrm{i}=1}^{\mathrm{N}} \frac{\partial \mathrm{R}_{\mathrm{i}, \text { th }}}{\partial \mathrm{R}_{\mathrm{sd}}}\left(\mathrm{R}_{\mathrm{i}, \text { th }}-\mathrm{R}_{\mathrm{i}, \exp }\right)=0
\end{array}\right.
$$

After some mathematical manipulations the above system of three equations is expressed as:

$$
\left\{\begin{array}{l}
\frac{1}{\mathrm{~K}} \sum_{\mathrm{i}=1}^{\mathrm{N}} \mathrm{EXP}_{\mathrm{i}}^{2}+\frac{\mathrm{R}_{\mathrm{sd}}}{\mathrm{V}_{\mathrm{d}}} \sum_{\mathrm{i}=1}^{\mathrm{N}} \mathrm{EXP}_{\mathrm{i}} \cdot \mathrm{V}_{\mathrm{gti}}=\sum_{\mathrm{i}=1}^{\mathrm{N}} \mathrm{EXP}_{\mathrm{i}} \mathrm{R}_{\mathrm{i}, \exp } \\
\frac{1}{\mathrm{~K}} \sum_{\mathrm{i}=1}^{\mathrm{N}} \mathrm{EXP}_{\mathrm{i}} \cdot \mathrm{V}_{\mathrm{gti}}+\frac{\mathrm{R}_{\mathrm{sd}}}{\mathrm{V}_{\mathrm{d}}} \sum_{\mathrm{i}=1}^{\mathrm{N}} \mathrm{V}_{\mathrm{gti}}^{2}=\sum_{\mathrm{i}=1}^{\mathrm{N}} \mathrm{R}_{\mathrm{i}, \exp } \mathrm{V}_{\mathrm{gti}}
\end{array}\right.
$$

After resolving the previous system, the expressions of $K$ and Rsd as function of $\theta$ are given by: 


$$
\left\{\begin{array}{l}
K(\theta)=\frac{a \cdot c-b^{2}}{d \cdot c-b \cdot e} \\
R_{s d}(\theta)=V_{d} \cdot \frac{a \cdot e-b \cdot d}{a \cdot c-b^{2}}
\end{array}\right.
$$

Where $\mathrm{a}, \mathrm{b}, \mathrm{c}, \mathrm{d}$ and e are given by:

$$
\begin{aligned}
& \mathrm{a}=\sum_{\mathrm{i}=1}^{\mathrm{N}} \mathrm{EXP}_{\mathrm{i}}^{2}, \mathrm{~b}=\sum_{\mathrm{i}=1}^{\mathrm{N}} \mathrm{EXP}_{\mathrm{i}} \cdot \mathrm{V}_{\mathrm{gti}} ; \mathrm{c}=\frac{\mathrm{R}_{\mathrm{sd}}}{\mathrm{V}_{\mathrm{d}}} \sum_{\mathrm{i}=1}^{\mathrm{N}} \mathrm{V}_{\mathrm{gti}}^{2} \\
& \mathrm{~d}=\sum_{\mathrm{i}=1}^{\mathrm{N}} \mathrm{EXP}_{\mathrm{i}} \mathrm{R}_{\mathrm{i}, \exp } ; \mathrm{e}=\sum_{\mathrm{i}=1}^{\mathrm{N}} \mathrm{R}_{\mathrm{i}, \exp } \mathrm{V}_{\mathrm{gti}}
\end{aligned}
$$

With the aim of the extraction of parameter $\theta$, we use an iterative technique which fond the optimal value of $\theta$ at the minimum of the RMSE:

$$
\mathrm{RMSE}=\sqrt{\frac{1}{\mathrm{~N}} \sum_{\mathrm{i}=1}^{\mathrm{N}}\left(\mathrm{R}_{\mathrm{i}, \exp }-\mathrm{R}_{\mathrm{i}, \text { th }}\right)^{2}}
$$

The algorithm of the proposed method to extract the series resistance, the mobility attenuation parameter is summarized in the Figure 2.

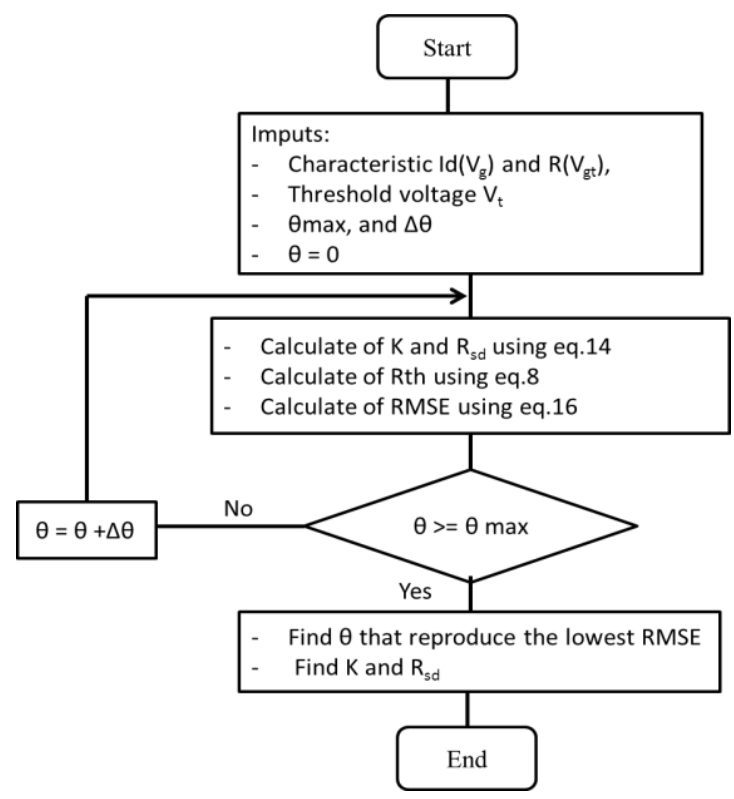

Figure 2. Flow chart of calculating parameters

\section{RESULTS AND DISCUSSION}

In order to validate the presented method, the MOSFET transistor measured in this work has the following parameters: channel width $\mathrm{W}=4 \mu \mathrm{m}$, gate oxide thickness tox $=5 \mathrm{~nm}$, channel length $\mathrm{L}=0.1 \mu \mathrm{m}$, and channel doping $\mathrm{Na}=10^{16} \mathrm{~cm}^{-3}$. The threshold voltage of this device is $\mathrm{V}_{\mathrm{t}}=0.2 \mathrm{~V}$. The proposed method was implemented in MATLAB.

Table 1 shows the extracted parameters using the proposed method obtained for this device and compared with two methods used the same device and the same exponential model of mobility. As it can be seen, the results obtained using the proposed method, have a good agreement with those previously published in the two works. The surface roughness amplitude is extracted using the (5). 
Table 1. Extraction parameters using the proposed method

\begin{tabular}{cccc}
\hline Parameter & $\mathrm{R}_{\mathrm{sd}}(\Omega)$ & $\theta\left(\mathrm{V}^{-1}\right)$ & $\Delta(\mathrm{nm})$ \\
\hline Proposed method & 108.82 & 0.36 & 0.28 \\
Method in [10] & 108.80 & 0.36 & 0.28 \\
Method in [4] & 107.53 & 0.36 & 0.31 \\
\hline
\end{tabular}

Figure 3 shows the plot of the RMSE versus the mobility degradation parameter, the value of $\Delta \theta$ used in this algorithme equal to 0.01 . As it can be seen, the optimal value of $\theta$ that reproduce the lowest value of $\mathrm{RMSE}=2.403$ is $0.36 \mathrm{~V}^{-1}$.

Figure 4 shows the experimental characteristic and the theoretical one of the resistance $\mathrm{R}$ versus $\mathrm{Vgt}$ at ohmic regime. The theoretical values of $\mathrm{R}$ are calculated by injecting the values of the extracted parameters using our method in the (8). It is clear that the calculated curve using the obtained parameters fit the experimental curve with accuracy.

The experimental characteristic of the drain current versus $\mathrm{V}_{\mathrm{g}}$ and the theoretical curve calculated from the extracted parameters and using the (6) are plotted in Figure 5. The application of the presented gives a best fit with experimental data in ohmic regime.

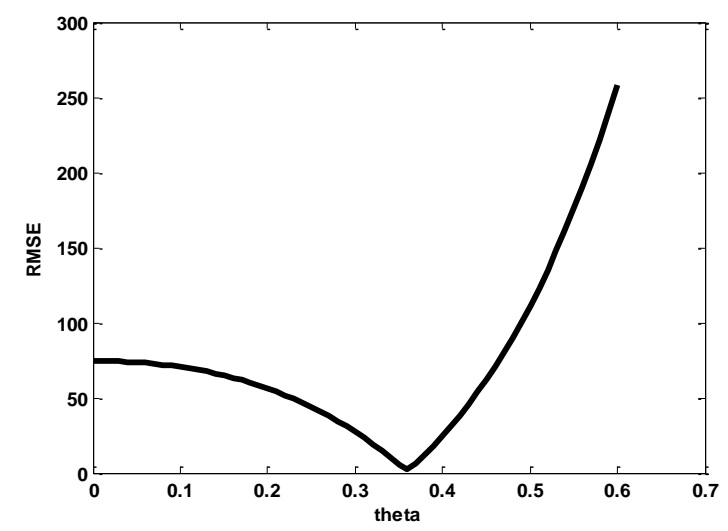

Figure 3. Plot of RMSE vs. theta

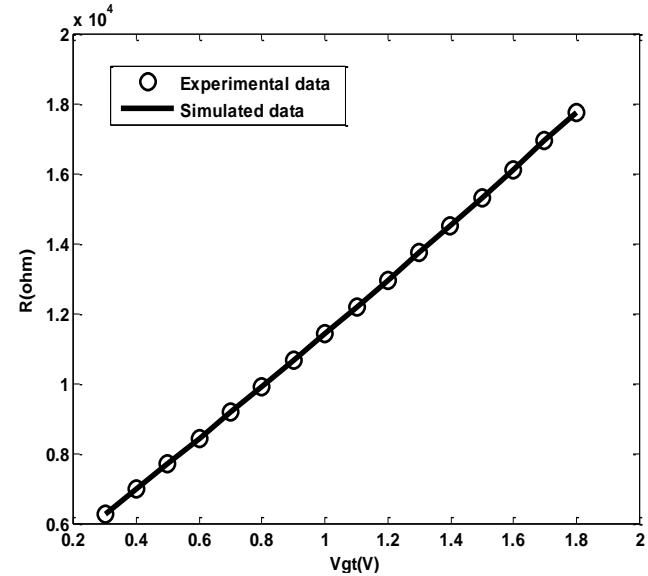

Figure 4. Plot of experimental and theoretical characteristics of R vs. Vgt

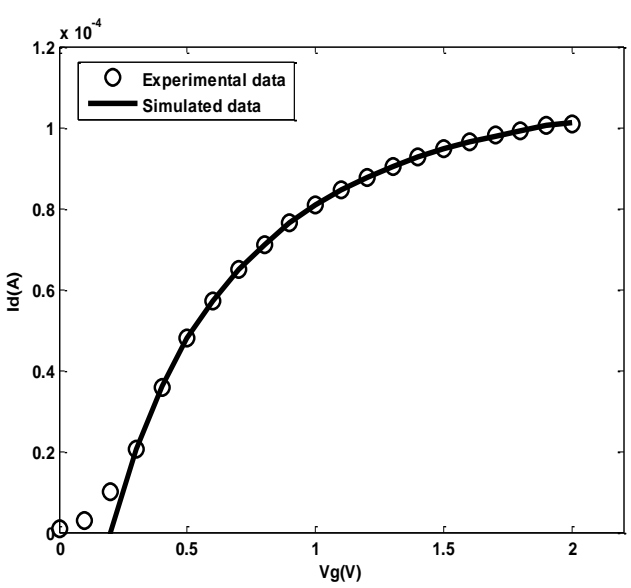

Figure 5. Plot of experimental and theoretical Id vs. Vg characteristics

\section{CONCLUSION}

In this paper, an iterative method for the extraction of the parasitic series resistance and the mobility attenuation parameters in MOSFET transistors was presented. This technique is based on the analytical expression of the series resistance and the mobility at low field using linear least squares method and solving 
a system of two equations as function mobility attenuation parameter. The mobility degradation parameter is determined iteratively as the one, that minimizes the RMSE value. The validation of this method using a single device shows a good agreement with experimental data. The obtained parameter values have been also compared with the parameters obtained for the same device using other methods available in the literature. Consistency between the results has been demonstrated.

\section{REFERENCES}

[1] A. Ortiz-Conde, J.J. Liou, W. Wong, F.J. García-Sánchez, "A simple method to extract the difference of the drain and source series resistances in MOSFETs", Electron. Lett. 30, pp. 1013-1015, 1994.

[2] A. Ortiz-Conde, F.J. García-Sánchez, J.J. Liou, "An improved method for extracting the difference between drain and source resistances in MOSFETs", Solid State Electron. 39, pp. 419-421, 1996.

[3] G. Niu, J.D. Cressler, S.J. Mathew, S. Subbanna, "A total resistance slope-based effectivechannel mobility extraction method for deep submicron CMOS technology", IEEE Trans. Electron Dev. 46, pp. 1912-1914, 1999.

[4] A. EL Abbassi, Y. Amhouche, E. Bendada, R. Rmaily and K. Rais. "Characterization of series resistance and mobility attenuation phenomena in short channel MOS transistors". Active and Passive Electronic Components, vol. 24, no1, pp. 13-22, 2001.

[5] G.S. Kar, S. Maikap, S.K. Banerjee, S.K. Ray, "Series resistance and mobility degradation factor in C-incorporated SiGe heterostructure p-type metal-oxide semiconductorfield-effect transistors", Semicond. Sci. Technol. 17, pp. 938-941, 2002.

[6] F.J. García-Sánchez, A. Ortiz-Conde, A. Cerdeira, M. Estrada, D. Flandre, J.J. Liou, "A method to extract mobility degradation and total series resistance of fully-depleted SOI MOSFETs", IEEE Trans. Electron Dev. 49, pp. 82-88, 2002.

[7] D.W. Lin, M.L. Cheng, S.W.Wang, C.C.Wu,M.J. Chen, "A constant-mobility method to enable MOSFET seriesresistance extraction”, IEEE Electron Dev. Lett. 28, pp. 1132-1134, 2007.

[8] W.P.N. Chen, P. Su, K.I. Goto, C.H. Díaz, "Series resistance and mobility extractionmethod in nanoscale MOSFETs", J. Electrochem. Soc. 156, pp. H34-H38, 2009.

[9] A. Ortiz-Conde, F.J. García Sánchez, J. Muci, D.C. Lugo Muñoz, A.D. Latorre Rey, C.-S. Ho, J.J. Liou, "Indirect fitting procedure to separate the effects of mobility degradation and source-and-drain resistance in MOSFET parameter extraction", Microelectron. Reliab. 49, pp. 689-692, 2009.

[10] J. Muci, D. Lugo Muñoz, A. Latorre Rey, A. Ortiz-Conde, F.J. García-Sánchez, C.-S. Ho, J.J. Liou, "A new integration-based procedure to separately extract series resistance and mobility degradation in MOSFETs", Semicond. Sci. Technol. 24, p. 105015, 2009.

[11] J. Muci, A. Latorre-Rey, F.J. García-Sánchez, D. Lugo-Muñoz, A. Ortiz-Conde, C. Ho, J.J. Liou, M.A. Pavanello, R. Doria, "Extraction of mobility degradation and source-anddrain resistance in MOSFETs", J. Integr. Circuits and Syst. 5, pp. 103-109, 2010.

[12] N. Maouhoub, K. Rais, "Characterization of series resistance and mobility degradationparameter and optimizing choice of oxide thickness in thin oxide N-channelMOSFET", Active and Passive Electron. Compon., p. 713129, 2011.

[13] Y.-H. Chang, Y.-J. Liu, "A self-consistent extraction procedure for source/drain resistance in MOSFETs", Microelectron. Reliab. 51, pp. 2049-2052, 2011.

[14] T. Chu, R.A. Vega, E. Alptekin, D. Guo, H. Shang, "Understanding short channel mobility degradation by accurate external resistance decomposition and intrinsic mobility extraction", J. Appl. Phys. 117, $064507,2015$.

[15] H. Xia, X. Ji, L. Ma, Y. Chen, F. Yan, "The comparison of various S/D series resistancesmethods for deeply submicron MOSFETs”, Electrochem. Soc. Trans. 60, pp. 963-968, 2014.

[16] J.-C. Guo, Y.-Z. Lo, "A new method for accurate extraction of source resistance and effective mobility in nanoscale multifinger nMOSFETs", IEEE Trans. Electron Dev. 62, pp. 3004-3011, 2015.

[17] A. Sucre-González, F. Zárate-Rincón, A. Ortiz-Conde, R. Torres-Torres, F.J. GarcíaSánchez, J. Muci, R.S. Murphy-Arteaga, "A DC method to extract mobility degradation and series resistance of multifinger microwave MOSFETs”, IEEE Trans. Electron Dev. 63, pp. 1821-1826, 2016.

[18] P.-H. Su, Y. Li, "Determination of source-and-drain series resistance in 16-nm-ga FinFET devices", IEEE Trans. Electron Dev. 62, pp. 1663-1667, 2015.

[19] S. Jun, H. Bae, H. Kim, J. Lee, S.-.J. Choi, D.H. Kim, D.M. Kim, "Dual-sweep combinational transconductance technique for separate extraction of parasitic resistances in amorphous thin film transistors", IEEE Electron Dev. Lett. 36, pp. 144-146, 2015.

[20] A. Cerdeira, M. Estrada, L.F. Marsal, J. Pallares, B. Iñiguez, "On the series resistance in staggered amorphous thin film transistors", Microelectron. Reliab. 63, pp. 325-335, 2016.

[21] A. Ortiz-Condeet al., "A review of DC extraction methods for MOSFET series resistance and mobility degradation model parameters", Microelectron. Rel., vol. 69, pp. 1-16, Feb. 2017.

[22] Rodolfo Rodriguez-Davila, Adelmo Ortiz-Conde , Senior Carlos Avila-Avendano , and Manuel A. QuevedoLopez, "A New Integration-Based Procedure to Extract the Threshold Voltage, the Mobility Enhancement Factor, and the Series Resistance of ThinFilm MOSFETs". IEEE Trans. Electron Dev, 66, pp. 2979-2985, 2019. 